\title{
Modeling Conditional Dependence of Stock Returns Using a Copula-based GARCH Model
}

\author{
Eun-Joo Lee ${ }^{1}$, Noah Klumpe ${ }^{1}$, Jonathan Vlk ${ }^{2}$ \& Seung-Hwan Lee ${ }^{2}$ \\ ${ }^{1}$ Department of Mathematics, Millikin University, Decatur, IL 62522, USA \\ ${ }^{2}$ Department of Mathematics, Illinois Wesleyan University, Bloomington, IL 61702, USA \\ Correspondence: Seung-Hwan Lee, Department of Mathematics, Illinois Wesleyan University, Bloomington, IL 61702, \\ USA. E-mail: slee2@iwu.edu
}

Received: September 25, 2016

Accepted: December 28, 2016 Online Published: February 13, 2017

doi:10.5539/ijsp.v6n2p32

URL: https://doi.org/10.5539/ijsp.v6n2p32

\begin{abstract}
Investigating dependence structures of stocks that are related to one another should be an important consideration in managing a stock portfolio, among other investment strategies. To capture various dependence features, we employ copula to overcome the limitations of traditional linear correlations. Financial time series data is typically characterized by volatility clustering of returns that influences an estimate of a stock's future price. To deal with the volatility and dependence of stock returns, this paper provides procedures of combining a copula with a GARCH model which leads to the construction of a multivariate distribution. Using the copula-based GARCH approach that describes the tail dependences of stock returns, we carry out Monte Carlo simulations to predict a company's movements in the stock market. The procedures are illustrated in two technology stocks, Apple and Samsung.
\end{abstract}

Keywords: copula, GARCH, dependence structure, stock return

\section{Introduction}

In stock portfolio management, the dependence structure of stocks should be of important consideration in order to make profit and minimize losses, which explains from how the stock markets are related in one way or another, under certain economic conditions, a company's stock price may rise or fall, as another company's stock price rises or falls: they move in tandem with each other. Sometimes, these companies' stock prices may be adversely affected. Such phenomena are frequently observed in competitive companies, such as Apple and Samsung in the technology market. Linear correlation is often used to describe such relationships between stocks. However, it cannot capture the non-linear dependence relationships. Linear correlation is unable to capture the dependence structure, especially when extreme events are highly dependent between lines of business, which is consequently misleading when estimating total required economic capital (Embrechts, McNeil \& Straumann, 1999).

Modeling dynamic dependence between stock returns with a multivariate distribution is a complex task, especially when returns follow a non-normal distribution. A copula is a function that binds univariate marginal distributions to produce a multivariate distribution, where various types of dependence can be represented (Sklar, 1959). It is well known that Financial data tends to exhibit tail dependence that describes the behavior during extreme events, that implies actual extreme events to happen more often than forecasted by the normal distribution (Bali, et al., 2009). When stock prices are correlated in the tail events, a large gain or loss will have an impact on future prices, and thus, these tail events should be taken into account when modeling. Copulas are useful tools to model multivariate distributions with various possible tail dependence features, since they can capture a wide range of tail dependence structures, which include symmetric and asymmetric structures (Joe, et al., 2010). To handle various types of dependencies of stock returns, this work employs elliptical and Archimedean copulas.

In addition to the dependence issue above, another important issue in modeling is that financial time series data tends to exhibit volatility clustering (i.e., conditional variance), which is often referred to as heteroscedasticity. If the volatility of returns is not constant over time, a copula model could lead to under or over-predictions. Inaccurate predictions can lead to a failure to reassure a gain or loss in the stock market. To filter the volatility clustering effect of the data, a GARCH (Generalized Autoregressive Conditional Heteroskedastic) model (Bollerslev, 1986) is used in our study. When returns are serially correlated, meaning that large or small returns tend to occur in clusters causing its series to have a period of increased or decreased variation, an ARCH model is useful(Engle, 1982; Bera \& Higgins 1993; Campbell, Lo \& MacKinlay, 1997). A GARCH model is a generalized ARCH model, where the error variance is specified by an ARMA 
(Autoregressive Moving Average) model (Bollerslev, 1986). GARCH models have been used in financial time series to deal with future conditional variances (Angelidis et al., 2004; Lu et al., 2014). In particular, a number of reports in the literature indicate that the most commonly used specification is $\operatorname{GARCH}(1,1)$. An example includes Hansen and Lunde (Hansen \& Lunde, 2005) that compared 330 GARCH-type models in the analysis of IBM returns, they found that there is no evidence that the $\operatorname{GARCH}(1,1)$ is inferior to other models.

In this paper, we fit the copula-based GARCH model to Apple and Samsung's weekly historical stock prices from the first week of January in 2010 to the last week of May of 2016. Apple Inc. and Samsung Electronics Co., Ltd. are two of the most notorious companies that compete in the technology industry. We obtain marginal distributions of Apple and Samsung stocks using a GARCH model to accommodate time-varying conditional volatility, and then combine with a copula to fit the dependence structure of the two stock returns. Goodness-of-fit tests show that compared to the other copulas considered, Student's t-copula for GARCH(1,1) well describes the dependence structure of Apple and Samsung stock returns. Based on the copula-based GARCH model, we perform Monte Carlo simulations to predict stock price movements of Apple and Samsung.

This paper is organized as follows: Section 2 provides a definition of a GARCH model, Section 3 gives an overview of copulas, and describes elliptical and Archimedean copulas used in our analysis. Section 4 presents data and its characteristics. Procedures of applying the copula-based GARCH model to data and numerical results are discussed in Section 5. Finally, Section 6 presents concluding remarks.

\section{GARCH Model}

For financial time series analysis, we often consider the log return changes as data. Denote it by $x_{t}$ at time $t$. The usual mean equation of $x_{t}$ is (Engle, 1982)

$$
x_{t}=E\left(x_{t} \mid \psi_{t-1}\right)+\varepsilon_{t},
$$

where $E\left(x_{t} \mid \psi_{t-1}\right)$ is the conditional mean of $x_{t}$ given $\psi_{t-1}$, which is the information at time $t-1$. In order to describe the time series dependence and the conditional mean, we combine $\operatorname{AR}(p)$ (autoregressive of order $p$ ) and $\operatorname{MA}(q)$ (moving average of order $q)$ to get a $\operatorname{ARMA}(p, q)$ model,

$$
x_{t}=\mu+\sum_{i=1}^{p} \phi_{i} x_{t-i}+\sum_{j=1}^{q} \theta_{j} \varepsilon_{t-j}+\varepsilon_{t},
$$

where $\phi_{i}$ and $\theta_{j}$ are parameters, $\varepsilon_{t}$ is the white noise $\left(0, \sigma^{2}\right)$, and $\mu$ is a constant which may not be included if the mean of $x_{t}$ is zero, and when $\phi_{1}=0$ and $\theta_{1}=0$, then $\operatorname{ARMA}(1,1)=\operatorname{MA}(1)=\operatorname{ARMA}(0,1)$ and $\operatorname{ARMA}(1,1)=\operatorname{AR}(1)$ $=$ ARMA(1,0), respectively. Despite many advantages of ARMA, such as modeling conditional mean, the use of this model could be limited due to an assumption of constant variance (homoscedasticity). This assumption is often violated since financial time series data generally exhibits non-constant variance (Tsay 2002), often referred to as heteroscedasticity. The ARCH (Autoregressive Conditional Heteroscedasticity) model introduced by Engle (1982) could explain volatility clustering and heavy-tailed financial returns. The GARCH model is a generalized ARCH model, where the error variance is specified by an ARMA (Autoregressive Moving Average) model (Bollerslev, 1986). The $\varepsilon_{t}$ terms in the ARMA mean equation above are called the innovations of the time series process. Engle (1982) defined them as an autoregressive conditional heteroscedastic process such that

$$
\varepsilon_{t}=\sigma_{t} \eta_{t}
$$

where $\eta_{t}$ is a sequence of i.i.d. $(0,1)$ random variables, and $\sigma_{t}^{2}=E\left(\varepsilon_{t}^{2} \mid \psi_{t-1}\right)$ is the conditional variance of the error that changes over time. The variance equation of $\operatorname{GARCH}(\mathrm{p}, \mathrm{q})$ model is then (Bollerslev,1986)

$$
\begin{gathered}
\varepsilon_{t}=\sigma_{t} \eta_{t} \\
\sigma_{t}^{2}=\alpha_{0}+\sum_{i=1}^{p} \alpha_{i} \varepsilon_{t-i}^{2}+\sum_{i=1}^{q} \beta_{i} \sigma_{t-i}^{2},
\end{gathered}
$$

where $\alpha_{0}>0, \alpha_{i} \geq 0, \beta_{j} \geq 0, p \geq 0, q \geq 0, c$ is a constant, and the innovation sequence $\varepsilon_{t}$ is a sequence of i.i.d. $(0,1)$ random variables. To model the marginal distribution of data, we use ARMA for the conditional mean and GARCH to address the conditional variance, called an ARMA-GARCH model, which accounts for conditional 
heteroscedasticity (time-variant variance) and conditional dependence. An example includes ARMA(1,1)-GARCH(1,1) with Student's t-distribution innovations (Bollerslev,1986)

$$
\begin{gathered}
x_{t}=\mu+\phi x_{t-1}+\theta \varepsilon_{t-1}+\varepsilon_{t}, \\
\varepsilon_{t}=\sigma_{t} \eta_{t}, \\
\sigma_{t}^{2}=\alpha_{0}+\alpha_{1} \varepsilon_{t-1}+\beta_{1} \sigma_{t-1}^{2} .
\end{gathered}
$$

Dependence structure is an important source of information in order to lock in gains and avoid losses when managing stock portfolios. Thus, it should be taken into account when developing a model. In the following section, we discuss how to model dependence structures of the financial time series filtered by the ARMA-GARCH model. Financial data is known to have a relatively high correlation in tail events. We use copula to model the margins with the dependence structure.

\section{Copula}

The statistical properties and applications of copulas have been extensively studied in many recent works (e.g., Frees and Valdez 1998; Breymann et al. 2003; Demarta and McNeil 2005; Trivedi and Zimmer 2005). A copula is a function that combines univariate marginal distributions to construct a joint distribution with a specific dependence structure. Thus, it provides an easy way to create multivariate probability distributions that have a wide range of dependence and tail behavior. Sklar's theorem (1959) states that if $F$ is a joint distribution function with marginal distributions, $F_{1}, \ldots, F_{n}$, then there exists a copula $C$ such that

$$
F\left(x_{1}, \ldots, x_{n}\right)=C\left(F_{1}\left(x_{1}\right), \ldots, F_{n}\left(x_{n}\right)\right)
$$

Note that $F_{i}\left(X_{i}\right), i=1, \ldots, n$, has a uniform distribution defined on the interval[0,1]. Therefore, the copula can be viewed as a multivariate function with standard uniform marginal distribution. Equation (1), the dependence structure depends only on the type of copula, not on the choice of marginal distribution. So a copula function allows flexibility in the choice of marginal distributions. Let $u_{i}$ be the observed value of $F_{i}\left(X_{i}\right)$. Then, for continuous and strictly increasing univariate marginals, the unique copula function is given by

$$
C\left(u_{1}, \ldots, u_{n}\right)=F\left(F_{1}^{-1}\left(u_{1}\right), \ldots, F_{n}^{-1}\left(u_{n}\right)\right)
$$

where $F_{1}^{-1}, \ldots, F_{n}^{-1}$ denote the quantile functions of the marginals, $F_{1}, \ldots, F_{n}$. From Sklar's theorem, any marginals can be used since a copula links marginals to their multivariate distribution, and it is independent of marginals as a measure of dependence. Hence, such a copula is constructed under the assumption that marginal distributions are known and can be consistently estimated from a given data. Copulas provide a way of isolating the peculiar behavior of an individual stock return's marginal distribution from the description of dependence structure. Thus, copula modeling fits well into a stock return framework due to the fact that each stock return has a different distributional characteristic.

The two classes of copulas that will be covered are elliptical and Archimedean copulas. Student's t-copula which belongs to the class of elliptical copulas is (Embrechts, McNeil, \& Straumann, 2002),

$$
C_{v, \Sigma}^{t}\left(u_{1}, \ldots, u_{n}\right)=t_{v, \Sigma}\left(t_{v}^{-1}\left(u_{1}\right), \ldots, t_{v}^{-1}\left(u_{n}\right)\right)
$$

where $t_{v, \Sigma}$ denotes a multivariate $t$ distribution with $v$ degrees of freedom and $\Sigma$ correlation coefficient matrix, and $t_{v}$ is the marginal distribution of $t_{v, \Sigma}$. Student's t-copula allows for joint extreme events in both tails. Archimedean copulas are in the form

$$
C\left(u_{1}, \ldots, u_{n}\right)=\varphi\left(\varphi^{-1}\left(u_{1}\right)+\cdots+\varphi^{-1}\left(u_{n}\right)\right)
$$

for some generator function $\varphi$ and its generalized inverse $\varphi^{-1}$ (Nelson, 2006). From (4), the Gumbel copula (Gumbel 
1960; Hougaard 1986) is obtained by $\varphi(u)=(-\log u)^{\theta}, \theta \geq 1$. The Clayton copula (Clayton 1978; Cook \& Johnson 1981) is constructed by $\varphi(t)=\frac{t^{-\theta}-1}{\theta}$ for $\theta>0$. The generator $\varphi(t)=-\log \left[\frac{e^{-\theta t}-1}{e^{-\theta}-1}\right] \quad \theta \neq 0$ leads to the Frank copula (Frank, 1979). In those copulas, the parameter $\theta$ in the generator function $\varphi$ exhibits a degree of tail dependence. In contrast to Student's t-copula, Archimedean copulas take into account a range of asymmetric dependence structures.
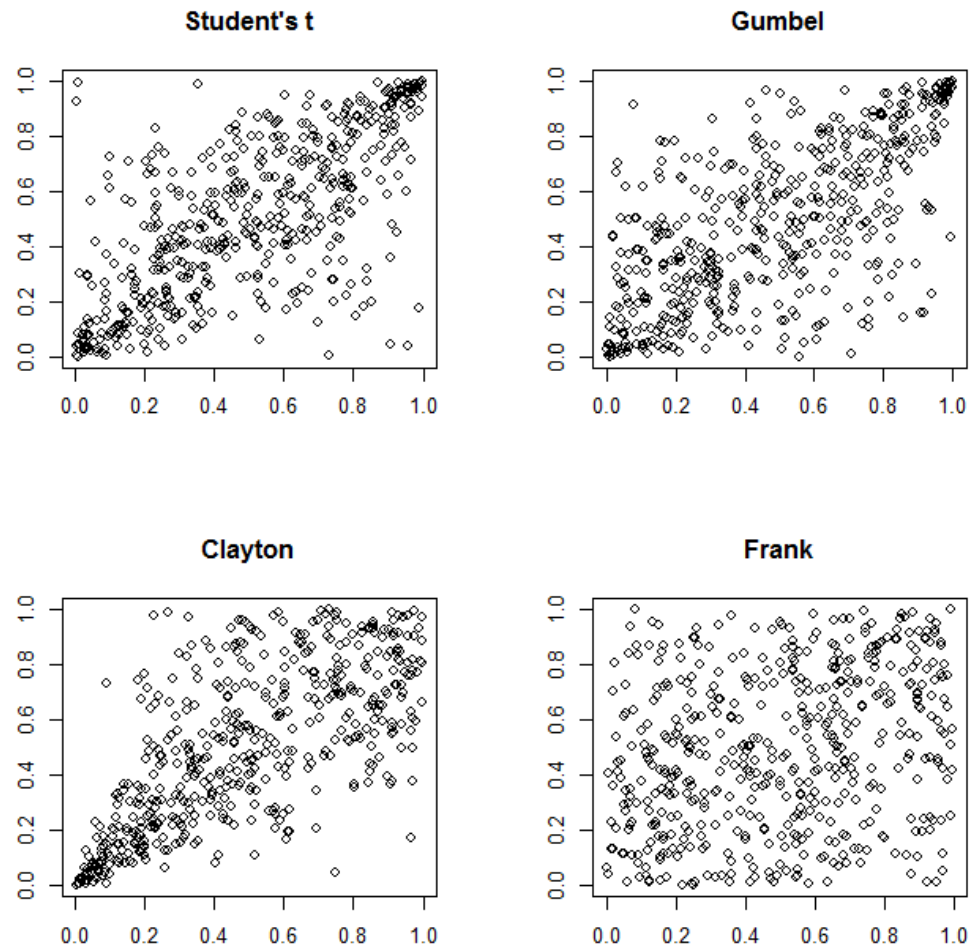

Figure 1. Scatter Plots of Student's t-copula, the Gumbel copula, the Clayton copula and the Frank copula

Figure 1 shows scatter plots of 500 pseudo observation for the copulas above (two variable case). From the plot of Student's t-copula, it can be seen that correlations tend to be present in extreme events, which are found in both upper and lower tails of the two variables. So Student's t-copula is suitable in modeling symmetric tail structure of data. The Gumbel copula has extreme events that are more correlated in the upper tail, while the Clayton copula has extreme events that are more correlated in the lower tail. This implies the Gumbel copula models upper tail dependence, and the Clayton copula captures lower tail dependence. So they are useful in modeling asymmetric tail dependence structures. It is seen that the Frank copula reveals no significant tail dependence.

\section{Data}

\subsection{History}

The Apple-Samsung business relationship began in the early 2000s when Apple first introduced its revolutionary mobile music playing device - the iPod. In the initial models, Apple used mini hard drives to store music, but soon found that there existed a much better option. At the time, Samsung was a producer of a faster and more reliable method of storing electronic information. Using flash memory technology, Apple was able to distribute its first flash-memory iPod device in 2005 after signing a deal with Samsung to lock in supply. For a brief period of time, Apple and Samsung worked very well together influencing the success of each other. Just two years later, Apple developed and released its first iPhone model in which most of its processing and memory was Samsung-made. Apple shifted its flash memory production away from Samsung and in 2009 and signed a deal with Toshiba Corporation. Apple found success in this move as in 2009, Samsung-made flash memory was in less than $10 \%$ of Apple products. In addition to flash memory and processors, touch 
screens were an essential aspect to the new generation of smartphones and Apple decided to stay away from Samsung on these component parts as well. The iPhone 4, which was released in October 2011, was its first model that didn't feature Samsung touch screens. Moving forward, as Apple was now developing its third-generation iPad, they realized that no one could match the production volume of Samsung's screens, which forced them to maintain ties with Samsung (Lessin, 2013).

Apple remained relatively close with Samsung as Samsung began to increase their smartphone and tablet business even further. Though in 2012, Apple won a \$1.05 billion lawsuit where it was found that Samsung infringed on six of Apple's patents. The results from the lawsuit left Apple's market position safe as well as their significant profit margins; however, it provided Samsung with new issues within their smartphone and tablet business (Vascellaro, 2012).

While the smartphone and tablet market is only a portion of Samsung's overall business, Apple wanted to maintain the look, feel, and aesthetic experience found in their smartphones and tablets. Samsung still beat Apple in 2014 smartphone volume, but Samsung saw its core mobile division profit margins decrease to 7\% (down from 18\% in 2013) as well as saw its mobile operating profits fall $42 \%$ (Cheng \& Lee, 2015).

Since Samsung's product line is much more extensive than that of Apple's, their overall stock price was less volatile throughout the time period of this study (2010-2016). Additionally, since Apple introduces new product lines every September, reoccurring volatility is expected based on how the market perceives the value of these new products. Though, when looking at small subsets of this time period, a correlation is found between the two companies' stocks. For example, both Apple and Samsung have a peak in percent change in stock price from February 2015 to May 2015 and a valley from August 2015 to October 2015. Thus, it may be beneficial to study a data set that covers a shorter period of time within this study to make the results more accurate. Nonetheless, both Apple and Samsung both reside in the technology sector and are equally affected by macroeconomic events, such as recessions, which provides a tangible economic relationship between the two technology giants.

\subsection{Marginal Time Series}

As indicated in Section 2, we use the rate of the log return changes as data. So, data that will be GARCH-filtered and copula-fitted are defined by

$$
x_{t+1}=\log \left(\frac{S_{t+1}}{S_{t}}\right)=\log S_{t+1}-\log S_{t},
$$

where $S_{t}$ is a stock's closing price at time $t$. Our data set includes weekly Apple and Samsung's closing prices from the first week of January in 2010 to the last week of May of 2016. Figure 2 shows the logarithm of weekly closing prices and returns of Apple and Samsung over the period of January 2010 through May 2016.

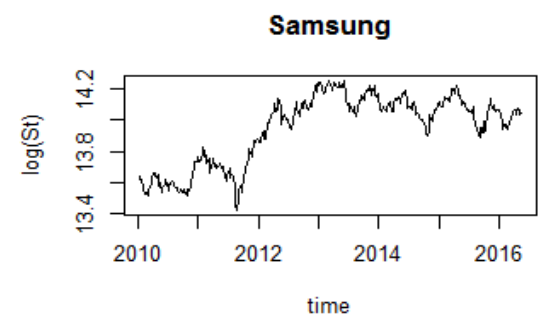

Samsung

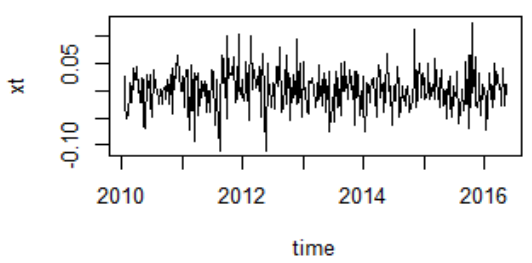

Apple

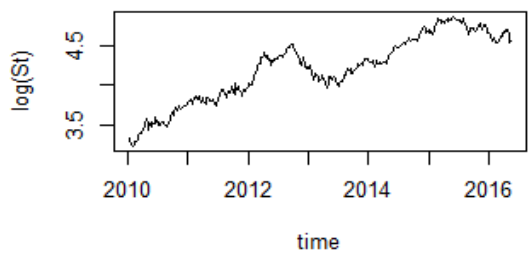

Apple

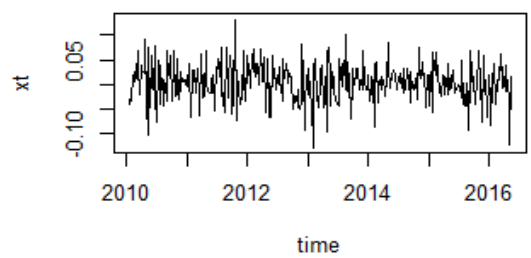

Figure 2. Apple and Samsung weekly closing prices and weekly returns, January 2000 - May 2016

It can be observed from Figure 2 that the returns have volatility clustering, which suggests that the GARCH model is the model to use. The conditional heteroscedasticity of the return series is also exhibited by ACF plots in Figure 3 . To further 
improve the objectivity of the graphical results, the Ljung-Box tests (Ljung \& Box, 1978), which formally assess autocorrelation of the series, were carried out at lags 1-19. P-values from the Ljung-Box tests are 0.0026 and 0.1415 for Apple and Samsung, respectively. Both Ljung-Box test values being close to zero and the graphical results suggest time varying conditional volatility, and this validates the use of the GARCH model.

Table 1 displays that the skewness for Samsung is 0.1223 , which implies the distribution is skewed to the right. On the other hand, it is observed that Apple is skewed to the left since it has a negative value of skewness. The kurtosis values greater than 3 imply that the returns have the heavy-tailed characteristic when compared to a normal distribution. The Jarque-Bera test results, along with the results of the skewness and kurtosis, indicate the non-normality of the returns. Note that GARCH model with Student's t-distribution innovations is appropriate to use for heavy-tailed time series (Bollerslev, 1987).
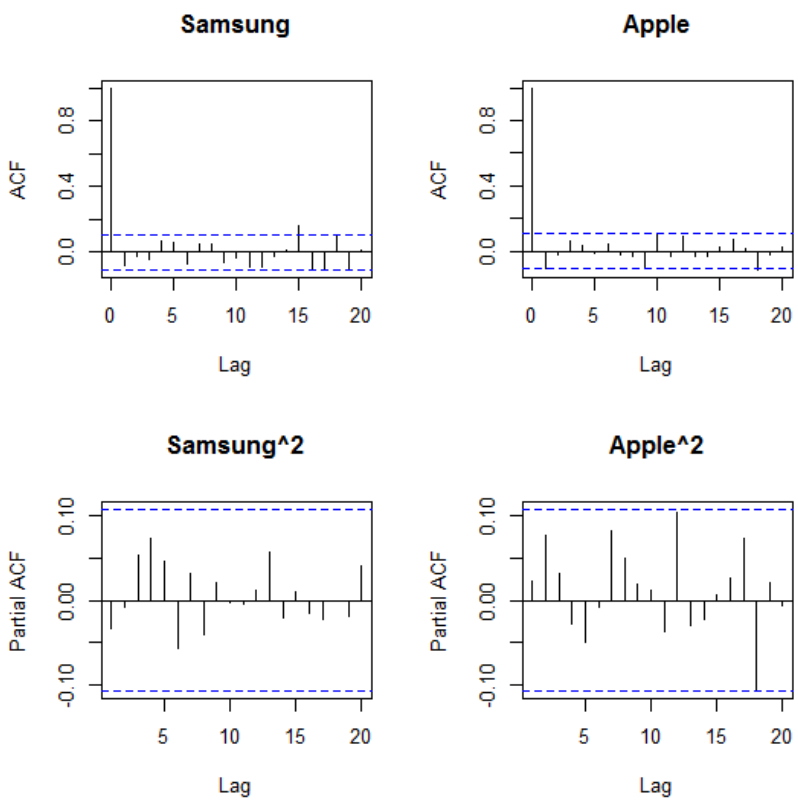

Figure 3. ACF of Apple and Samsung returns and squared returns, January 2000 - May 2016

Table 1. Summary statistics of the returns

\begin{tabular}{lll}
\hline & Samsung & Apple \\
\hline Min & -0.1111 & -0.1281 \\
Max & 0.1265 & 0.1320 \\
Mean & 0.0013 & 0.0037 \\
SD & 0.0359 & 0.0393 \\
Skewness & 0.1223 & -0.1722 \\
Kurtosis & 3.6712 & 3.3485 \\
Jarque-Bera p-value & 0.0231 & 0.0331 \\
\hline
\end{tabular}

\section{Procedures and Results}

Since the mean and the variance of the returns are correlated, the ARMA and GARCH models are used to deal with the serial correlation in the conditional mean and variance respectively. The Akaike Information Criteria (AIC) is used for the comparison of the models considered. Based on AIC, the ARMA(1,1) with Student's t-distribution innovations appears to be the most suitable model for the conditional mean, when compared to other specifications of $p$ and $q$. We also select $\operatorname{GARCH}(1,1)$ that accounts for the time-varying volatility of the series. The difference of the GARCH( $p, q)$ 
AIC values in consideration is quite small, so we have chosen the model with less parameters. Note that GARCH(1,1) is the most used heteroscedastic model in financial time series (Bera \& Higgins 1993). Thus, ARMA(1,1)-GARCH(1,1) with Student's t-innovations, which provides the global fit to all variables, is used for the marginal distribution that is associated with copula. Table 2 presents the estimates of the ARMA $(1,1)-\operatorname{GARCH}(1,1)$ parameters obtained by maximum likelihood method. AIC results for the model are also summarized in Table 2.

Table 2. Parameter estimates for ARMA(1,1)-GARCH(1,1) with Student's t-innovations

\begin{tabular}{lcccccc}
\hline Variables & $\mu$ & $\Phi$ & $\theta$ & $\alpha$ & $\beta$ & AIC \\
\hline Samsung & 0.0011 & -0.0776 & -0.0119 & 0.0093 & 0.9488 & -3.7969 \\
\hline Apple & 0.0040 & 0.1693 & -0.2763 & 0.0533 & 0.8677 & -3.6239 \\
\hline
\end{tabular}

From expression (1) in Section 3, copula has standard uniform marginal distribution. To obtain such margins, we standardize the residuals from $\operatorname{ARMA}(1,1)-\operatorname{GARCH}(1,1)$, and then convert to uniform $(0,1)$ samples using the probability integral transformation method. To model dependence structure with these uniform samples, Student's t-copula, the Gumbel copula, the Clayton copula and the Frank copula are utilized.

Lastly, to check the adequacy of copula based ARMA(1,1)-GARCH(1,1) for the observed data, we perform goodness of fit tests that will measure how well the model fits the data (Genest et al. 2009). The procedures are based on the comparison of the empirical copula and the corresponding theoretical copula obtained under the null hypothesis, where the empirical copula is copula (Deheuvels 1979) defined as

$$
\hat{C}(u)=\frac{1}{m+1} \sum_{j=1}^{m} I\left\{Z_{j 1} \leq u_{1}, \ldots, Z_{j n} \leq u_{n}\right\},
$$

where $Z_{j i}$ 's $, i=1, \ldots, n$, represent the pseudo-samples defined as

$$
Z_{j i}=\left(\frac{r_{j 1}}{m+1}, \ldots, \frac{r_{j n}}{m+1}\right),
$$

where $r_{j i}$ is the rank of the observation $X_{i}$. The Cramer-von Mises statistic by Genest et al. (2009) is

$$
\hat{T}=m \int_{[0,1]^{d}}\{\hat{C}(u)-C(u)\}^{2} d \hat{C}(u),
$$

where $C(u)$ is a copula associated with the estimates of parameters. This statistic, $\hat{T}$, measures the squared deviations of the empirical copula from the theoretical copula of the corresponding fit. Thus a small value indicates that the model has a good fit. Based on $T$ in (6), Student's t-copula with 52 degrees of freedom for ARMA(1,1)-GARCH(1,1) is chosen to best describe the data, with a p-value of 0.2651. The Gumbel, Clayton and Frank copulas fit the data with p-values of $0.0035,0.1124$ and 0.0500 , respectively. The results show that the two stock returns, Apple and Samsung, show symmetrical tail dependence structures well-described by Student's t-copula, when compared to other copulas that have asymmetric tail features.

Using the copula chosen, we perform a Monte Carlo simulation that generates 200,000 simulated observations for Apple and Samsung to evaluate the performance of the return series. In the simulation algorithm, we use estimated parameters of the copula-based ARMA(1,1)-GARCH(1,1) model and Kendall's rank correlation coefficients as inputs. The following steps outline the simulation scheme:

Step 1. Obtain ARMA(1,1)-GARCH(1,1) standardized residuals.

Step 2. Transform the standardized residuals to Uniform $(0,1)$ samples.

Step 3. Fit the copula-based ARMA(1,1)-GARCH(1,1) to the transformed data.

Step 4. Generate 200,000 simulated observations of Apple and Samsung. Let $U_{1}$ and $U_{2}$ represent Apple and Samsung, respectively.

Step 5. Obtain $F_{1}^{-1}\left(U_{1}\right)$ and $F_{2}^{-1}\left(U_{2}\right)$ to transform the uniformly distributed values back into the original units. 
Step 6. Obtain n-step-ahead forecasts at a specified level. The forecast is based on the previous GARCH(1,1) variance.

Table 3 presents the rate of return estimates using Student's t-copula which is chosen as the best model for Apple and Samsung filtered by ARMA $(1,1)-G A R C H(1,1)$. We estimate the return in terms of percentage. The results in Table 3 present forecasts of the return rate in terms of percentage for the future periods from the first week denoted by ' $\mathrm{w} 1$ ' to the tenth week denoted by 'w10' after the last week of May in 2016 (i.e. the time of the last data point). We start with the first week of June 2016 (w1) due to our sample data spanning from the first week of January 2010 through the last week of May 2016. The results consist of the first (Q1), second (Q2) and third (Q3) quartiles of the distribution of 200,000 simulated observations. To report the results with a high, a median and a low estimate, we divide the 200,000 simulated observations into four equal groups, which leads to quartiles. Table 4 presents weekly predicted and actual prices calculated from Table 3. It is worth mentioning that all the actual prices in Table 4 are close to Q2 overall, bounded by Q1 and Q3. For instance, it is predicted that from the relationships with Samsung, Apple moves 0.427\% (w1:Q2, predicted $50^{\text {th }}$ percentile) to $\$ 97.81$ for the first week of June 2016 , with a high increase (w1:Q3, predicted $75^{\text {th }}$ percentile) of $2.513 \%$ and a $1.657 \%$ decline (w1:Q1, predicted $25^{\text {th }}$ percentile). Note that the actual Apple stock price for the last week of May $(5 / 30 / 2016)$ was $\$ 97.92$, and the first week of June (6/6/2016) was \$98.83. Based on the same model, Samsung has a median of $0.095 \%$ increase (w1:Q2), with a high increase of $2.060 \%$ (w1:Q3) and a low estimate of $-1.865 \%$ (w1:Q1) for the first week of June 2016.

Table 3. 10-step-ahead forecast (\%) for the Apple and Samsung returns

\begin{tabular}{lllllllllllll}
\hline Variable & Quartile & w1 & w2 & w3 & w4 & w5 & w6 & w7 & w8 & w9 & w10 \\
\hline Samsung & Q1 & -1.865 & -1.849 & -1.845 & -1.844 & -1.844 & -1.844 & -1.844 & -1.843 & -1.843 & -1.843 \\
& Q2 & 0.095 & 0.111 & 0.115 & 0.116 & 0.116 & 0.116 & 0.116 & 0.116 & 0.116 & 0.116 \\
& Q3 & 2.068 & 2.084 & 2.087 & 2.088 & $2 . .088$ & 2.088 & 2.088 & 2.088 & 2.088 & 2.087 \\
\hline Apple & Q1 & -1.657 & -1.689 & -1.689 & -1.688 & -1.688 & -1.688 & -1.687 & -1.687 & -1.687 & -1.686 \\
& Q2 & 0.427 & 0.394 & 0.394 & 0.394 & 0.394 & 0.394 & 0.394 & 0.394 & 0.394 & 0.394 \\
& Q3 & 2.513 & 2.479 & 2.479 & 2.479 & 2.479 & 2.478 & 2.478 & 2.478 & 2.478 & 2.477 \\
\hline
\end{tabular}

Table 4. Comparison of predicted and actual price, Apple and Samsung

\begin{tabular}{lllllll}
\hline Variable & Quartile/ & w1 & w2 & w3 & w4 & w5 \\
& Actual & & & & & \\
\hline Samsung & Q1 & KRW 1,350,353 & KRW 1,325,385 & KRW 1,300,932 & KRW 1,276,942 & KRW 1,253,396 \\
& Q2 & KRW 1,377,323 & KRW 1,378,852 & KRW 1,380,437 & KRW 1,382,039 & KRW 1,383,642 \\
& Q3 & KRW 1,404,472 & KRW 1,433,741 & KRW 1,463,663 & KRW 1,494,224 & KRW 1,525,424 \\
& Actual & KRW 1,404,995 & KRW 1,424,981 & KRW 1,398,999 & KRW 1,466,000 & KRW 1,460,000 \\
\hline Apple & Q1 & $\$ 95.78$ & $\$ 94.16$ & $\$ 92.57$ & $\$ 91.01$ & $\$ 89.47$ \\
& Q2 & $\$ 97.81$ & $\$ 98.19$ & $\$ 98.58$ & $\$ 98.97$ & $\$ 99.36$ \\
& Q3 & $\$ 99.84$ & $\$ 102.31$ & $\$ 104.85$ & $\$ 107.45$ & $\$ 110.11$ \\
& Actual & $\$ 98.83$ & $\$ 95.33$ & $\$ 93.40$ & $\$ 95.89$ & $\$ 96.68$ \\
\hline
\end{tabular}


Table 5. Continued

\begin{tabular}{lllllll}
\hline Variable & Quartile/ & w6 & w7 & w8 & w9 & w10 \\
& Actual & & & & & \\
\hline Samsung & Q1 & KRW 1,230,283 & KRW 1,207,597 & KRW 1,185,341 & KRW 1,163,495 & KRW 1,142,052 \\
& Q2 & KRW 1,385,247 & KRW 1,386,854 & KRW 1,388,463 & KRW 1,390,073 & KRW 1,391,686 \\
& Q3 & KRW 1,557,275 & KRW 1,589,791 & KRW 1,622,985 & KRW 1,656,873 & KRW 1,691,452 \\
& Actual & KRW 1,518,000 & KRW 1,516,000 & KRW 1,539,000 & KRW 1,561,000 & KRW 1,545,000 \\
\hline Apple & Q1 & $\$ 87.96$ & $\$ 86.48$ & $\$ 85.02$ & $\$ 83.58$ & $\$ 82.18$ \\
& Q2 & $\$ 99.75$ & $\$ 100.14$ & $\$ 100.54$ & $\$ 100.93$ & $\$ 101.33$ \\
& Q3 & $\$ 112.84$ & $\$ 115.64$ & $\$ 118.50$ & $\$ 121.44$ & $\$ 124.45$ \\
& Actual & $\$ 98.78$ & $\$ 98.66$ & $\$ 104.21$ & $\$ 107.48$ & $\$ 108.15$ \\
\hline
\end{tabular}

\section{Concluding Remarks}

A copula is a function that links a multivariate distribution to their univariate marginal distributions in such a way that allows various types of dependence to be represented. Thus, copula modeling enables us to explore the impact of dependencies between different stocks on their rate of returns. To deal with the complexities of the marginal distributions, such as mean and variance changing over time, we employed time series models, ARMA $(1,1)$ for the mean model and $\operatorname{GARCH}(1,1)$ for the variance model. The copula-based $\operatorname{ARAM}(1,1)-\operatorname{GARCH}(1,1)$ model developed in this work accounts for some important issues in financial markets, such as skewness, volatility clustering, and conditional dependence. We find that Student's t-copula, which has a symmetric tail dependence structure, with ARMA(1,1)-GARCH(1,1) fits the data well. Using this copula-based ARMA(1,1)-GARCH(1,1) model that considers the relationship of Apple and Samsung, we simulated a weekly return rate of those companies to measure forecasted performance. Our study also suggests some issues for future research. For example, the best fit was found for Student's t copula with 52 degrees of freedom, which suggests a Gaussian copula. The use of a Gaussian copula will observe the consequence of ignoring extreme events. Unlike Student's t-copula, a Gaussian copula does not allow for extreme events to be dependent, and thus underestimates the effects of extreme events in the heavy tails. For GARCH-filtered data that may have correlation structures with high dimensions, we can consider vine copulas (Aas, et al., 2009; Joe, 1996), which is recently developed for multivariate data, instead of elliptical or Archimedean copulas. Since vine copulas allow heterogeneous pairwise dependence of variables, it could be more flexible in high-dimensional dependence modeling. The methodology developed in this work can also give a reliable way to calculate Value at Risk, often denoted by VaR in the area of risk management, to quantify gains or losses on a portfolio that contains several variables.

\section{References}

Aas, K., Czado, C., Frigessi, A., \& Bakken, H. (2009). Pair-Copula Constructions of Multiple Dependence. Insurance: Mathematics and Economics, 44, 182-198. https://doi.org/10.1016/j.insmatheco.2007.02.001

Angelidis, T., Benos, A. \& Degiannakis, S. (2004). The Use of GARCH Models in VaR Estimation, Statistical Methodology, 1, 105-128. https://doi.org/10.1016/j.stamet.2004.08.004

Bali, T., Demirtas, K., \& Levy, H. (2009), Is There an Intertemporal Relation between Downside Risk and Expected Returns? Journal of Financial and Quantitative Analysis, 44(4), 883-909.

Bera, A., \& Higgins, M. (1993), ARCH models: Properties, Estimation and Testing, Journal of Economic Surveys, 7 , 305-366. https://doi.org/10.1111/j.1467-6419.1993.tb00170.x

Bollerslev, T. (1986), Generalized Autoregressive Conditional Heteroskedasticity, Journal of Econometrics, 31, 307-327.

Breymann, W., Dias, A., \& Embrechts, P. (2003). Dependence Structures for Multivariate High-Frequency Data in Finance. Quantitative Finance, 3, 1-14. https://doi.org/10.1080/713666155

Campbell, J. Y., Lo, A. W., \& MacKinlay, A. C. (1997). The Econometrics of Financial Markets. Princeton, New Jersey: Princeton University Press. 
Cheng, J. \& Lee, M. -J. (2015). Samsung Questions its Game Plan -South Korean Giant does some Soul-Searching as Smartphone Sales Disappoint. Wall Street Journal.

Clayton, D. G. (1978). A Model for Association in Bivariate Life Tables and its Application in Epidemiological Studies of Familial Tendency in Chronic Disease Incidence, Biometrika, 65, 141-151. https://doi.org/10.1093/biomet/65.1.141

Cook, R. D., \& Johnson, M. E. (1981). A family of Distributions for Modeling Non- Elliptically Symmetric Multivariate Data, Journal of the Royal Statistical Society, Series B, 43, 210-218.

Deheuvels, P. (1979). La Fonction de dependance empirique et ses proprieties: un test non parametrique d'independence. Bulletin de la Classe Des Sciences. Academie Royal de Belgique, 65, 274-292

Demarta, S., \& McNeil, A. (2005). The t Copula and related Copulas. International Statistical Review, 73, 111-129. https://doi.org/10.1111/j.1751-5823.2005.tb00254.x

Embrechts, P., McNeil, A., \& Straumann, D. (2002). Correlation and Dependency in Risk Management: Properties and Pitfall. In Risk Management: Value at Risk and Beyond, ed. M.A.H. Dempster, Cambridge University Press, Cambridge, 176-223. https://doi.org/10.1017/CBO9780511615337.008

Engle, R. (1982). Autoregressive Conditional Heteroskedasticity with Estimates of the Variance of United Kingdom Inflation, Econometrica, 50, 987-1007. https://doi.org/10.2307/1912773

Frank, M. J. (1979). On The Simultaneous Associativity of $\mathrm{F}(\mathrm{x}, \mathrm{y})$ and.x+y-F(x,y). Aequationes Mathematicae, 19, 194-226. https://doi.org/10.1007/BF02189866

Frees, E., Carriere, J., \& Valdez, E. (1996) . Annuity Valuation with Dependent Mortality, Journal of Risk and Insurance, 63, 229-261. https://doi.org/10.2307/253744

Genest, C., Remillard, B. \& Beaudoin, D. (2009) . Goodness-of-Fit for Copulas; A Review and Power Study. Insurance, Mathematics \& Economics, 44, 199-213. https://doi.org/10.1016/j.insmatheco.2007.10.005

Gumbel, E. J. (1960). Bivariate Exponential Distributions, Journal of American Statistical Association, 55, 698-707.

Hansen, P., \& Lunde, A. (2005) . A Forecast Comparison of Volatility Models: Does Anything Beat a GARCH(1,1)?, Journal of Applied Economics, 20, 873-889. https://doi.org/10.1002/jae.800

Hougaard, P. (1986). A class of Multivariate Failure Time Distributions. Biometrika, 73, 671-678.

Joe, H. (1996). Families of m-variate Distributions with Given Margins and m(m-1)/2 Bivariate Dependence Parematers. In L. Ruschendorf and B. Schweizer and M.D. Taylor (Ed.. Distributions with Fixed Marginals and Related Topics.

Joe, H., Li, H., \& Nikoloulopoulos, A. K. (2010). Tail dependence functions and vine copulas. Journal of Multivariate Analysis, 101, 252-270. https://doi.org/10.1016/j.jmva.2009.08.002

Lessin, J. E., Luk, L., \& Osawa, J. (2013). Apple Finds it Difficult to Divorce Samsung, Wall Street Journal.

Ljung, G. M., \& Box, G. E. (1978). On a Measure of a Lack of Fit in Time Series Models, Biometrika, 65, 297-303

Lu, X., Lai, K. \& Liang, L. (2014). Portfolio Value-at-Risk Estimation in Energy Futures Markets with Time-varying Copula-GARCH Model. Annals of Operations Research, 219, 333-357.

Nelsen, R. (2006). An Introduction to Copulas, New York, Springer-Verlag, 1-270.

Sklar, A. (1959). Functions de Repartition a n Dimensions et leurs Merges. Publication of the Institute of Statistics, University of Paris, 8, 229-231.

Trivedi, P. K., \& Zimmer, D. M. (2005). Copula Modeling: An Introduction for Practitioners, Foundations and Trends in Economics, 1, 1-111. https://doi.org/10.1007/s10479-011-0900-9

Tsay, R. S. (2002). Analysis of Financial Time Series, Wiley. https://doi.org/10.1002/0471264105

Vascellaro, J. E. (2012). Apple Wins Big in Patent Case -Jury Finds Samsung Mobile Devices Infringes Six Apple Patents, Awards \$1.05 Billion in Damages, Wall Street Journal.

Vlaar, P., \& Palm, F. (1993). The Message in Weekly Exchange Rates in the European Monetary System, Mean Reversion, Conditional Heteroscedasticity, and Jumps, Journal of Business and Economic Statistics, 11, 351-360.

\section{Copyrights}

Copyright for this article is retained by the author(s), with first publication rights granted to the journal.

This is an open-access article distributed under the terms and conditions of the Creative Commons Attribution license (http://creativecommons.org/licenses/by/4.0/). 